\title{
Potential clinical value and putative biological function of miR-122-5p in hepatocellular carcinoma: A comprehensive study using microarray and RNA sequencing data
}

\author{
DONG-YUE WEN ${ }^{1}$, JIA-CHENG HUANG ${ }^{2}$, JIE-YU WANG ${ }^{2}$, WEN-YA PAN ${ }^{2}$, \\ JIANG-HUI ZENG ${ }^{2}$, YU-YAN PANG ${ }^{2}$ and HONG YANG ${ }^{1}$ \\ Departments of ${ }^{1}$ Medical Ultrasonics and ${ }^{2}$ Pathology, The First Affiliated Hospital of Guangxi \\ Medical University, Nanning, Guangxi Zhuang Autonomous Region 530021, P.R. China
}

Received July 14, 2017; Accepted December 12, 2017

DOI: $10.3892 / \mathrm{ol} .2018 .9523$

\begin{abstract}
In order to determine the diagnostic efficacy of microRNA (miR)-122-5p and to identify the potential molecular signaling pathways underlying the function of miR-122-5p in hepatocellular carcinoma (HCC), the expression profiles of data collected from The Cancer Genome Atlas (TCGA), Gene Expression Omnibus (GEO) and literature databases were analyzed, along with any associations between clinicopathological characteristics and the diagnostic value of miR-122-5p in HCC. The intersection of 12 online prediction databases and differentially expressed genes from TCGA and GEO were utilized in order to select the prospective target genes of miR-122-5p in HCC. Gene Ontology (GO), Kyoto Encyclopedia of Genes and Genomes (KEGG) and protein-protein interaction network (PPI) analyses were subsequently performed based on the selected target genes. The average expression level of miR-122-5p was decreased in HCC patients compared with controls from TCGA database $(\mathrm{P}<0.001)$, and the downregulation of miR-122-5p was significantly associated with HCC tissues $(\mathrm{P}<0.001)$, tumor vascular invasion $(\mathrm{P}<0.001)$, metastasis $(\mathrm{P}=0.001)$, sex $(\mathrm{P}=0.006)$, virus infection status $(\mathrm{P}=0.001)$ and tissue (compared with serum; $\mathrm{P}<0.001)$ in cases
\end{abstract}

Correspondence to: Professor Hong Yang, Department of Medical Ultrasonics, The First Affiliated Hospital of Guangxi Medical University, 6 Shuangyong Road, Nanning, Guangxi Zhuang Autonomous Region 530021, P.R. China

E-mail: yanghonggx@163.com

Abbreviations: HCC, hepatocellular carcinoma; TCGA, The Cancer Genome Atlas; GEO, Gene Expression Omnibus; GO, Gene Ontology; KEGG, Kyoto Encyclopedia of Genes and Genomes; PANTHER, Protein Analysis Through Evolutionary Relationships; PPI, and protein-protein interaction network; ROC, receiver operating characteristic; AUC, area under the curve; TP, true positive; $\mathrm{FP}$, false positive; $\mathrm{TN}$, true negative; $\mathrm{FN}$, false negative

Key words: microRNA-122-5p, hepatocellular carcinoma, diagnosis, bioinformatics analysis from the GEO database. The pooled sensitivity and specificity for miR-122-5p to diagnose HCC were 0.60 [95\% confidence interval (CI), 0.48-0.71] and 0.81 (95\% CI, 0.70-0.89), respectively. The area under the curve (AUC) value was $0.76(95 \%$ CI, 0.72-0.80), while in Meta-DiSc 1.4, the AUC was 0.76 $\left(Q^{*}=0.70\right)$. The pooled sensitivity and specificity were 0.60 (95\% CI, 0.57-0.62) and 0.79 (95\% CI, 0.76-0.81), respectively. A total of 198 overlapping genes were selected as the potential target genes of miR-122-5p, and 7 genes were defined as the hub genes from the PPI network. Cell division cycle 6 (CDC6), minichromosome maintenance complex component 4 (MCM4) and MCM8, which serve pivotal functions in the occurrence and development of HCC, were the most significant hub genes. The regulation of cell proliferation for cellular adhesion and the biosynthesis of amino acids was highlighted in the GO and KEGG pathway analyses. The downregulation of miR-122-5p in HCC demonstrated diagnostic value, worthy of further attention. Therefore, miR-122-5p may function as a tumor suppressor by modulating genome replication.

\section{Introduction}

Ranking fifth most common malignancies, hepatocellular carcinoma (HCC) is diagnosed in $>500,000$ individuals worldwide (2011) (1). With an increasing incidence, HCC has become the third most common cause of cancer-associated mortality worldwide (2010) (2). The majority of patients with HCC exhibit aggressive symptoms upon diagnosis and the median 1-year survival rate is $<30 \%(3,4)$. Traditionally, HCC is detected using biomarkers, including $\alpha$-fetoprotein $(5,6)$, with $39-65 \%$ sensitivity and 76-94\% specificity (7). Several biomarkers, such as miR-101-1, miR-221, miR-638 can be used as diagnostic and prognostic indicators of HCC (8-10). However, there are still a number of biomarkers that may be biologically relevant for the development of HCC. Therefore, a more efficient biomarker with improved accuracy is urgently required.

MicroRNAs (miRNAs) are small non-coding RNA molecules with an approximate length of 23 nucleotides. miRNAs serve as post-transcriptional regulators of gene expression by binding to the 3 '-untranslated region of mRNA $(11,12)$. 
Previous studies have demonstrated that the dysregulation of specific miRNAs contributes to the progression of hepatocarcinogenesis $(13,14)$. Therefore, these molecules may represent promising biomarkers for the diagnosis of HCC. A previous study demonstrated that miRNA (miR)-122-5p was downregulated in patients with $\mathrm{HCC}(15)$; however, its molecular mechanism and diagnostic significance remain to be elucidated.

The Cancer Genome Atlas (TCGA; https://cancergenome. nih.gov/) is a program, which contains RNA sequencing (RNA-Seq) data on 32 types of tumor without limitations or restrictions by the National Cancer Institute (NCI) and the National Human Genome Research Institute (NHGRI). The Gene Expression Omnibus (GEO; https://www.ncbi.nlm.nih. gov/geo/) is an online public resource provided by the National Center for Biotechnology Information (NCBI) in 2000. The GEO covers ample gene chip expression data, as well as data from non-chip technologies, such as serial analysis of gene and mass spectrometry. GEO provides large quantities of high-throughput data and is one of the most comprehensive public gene expression data resources available currently.

Therefore, the present study examined the accuracy of miR-122-5p in distinguishing HCC patients from healthy controls based on data collected from TCGA and GEO. The association between distinct clinicopathological features and the expression of miR-122-5p was also investigated. To further clarify the regulatory mechanism of miR-122-5p in HCC, subsequent bioinformatic analyses were conducted. The present study aims to perform a quantitative evaluation of the association between miR-122-5p in HCC and pathophysiological processes, specifically investigating the type of genes and signaling pathways that are associated with downregulated miR-122-5p levels in HCC specimens.

\section{Materials and methods}

Collection of TCGA and GEO data. TCGA provided miRNA raw sequencing data from $370 \mathrm{HCC}$ cases and 50 adjacent liver tissue samples. The publicly available RNA-Seq data were downloaded directly from the TCGA launch data portal via bulk download of the liver hepatocellular carcinoma (LIHC), RNASeqV2 (data type) and level 3 (data level) cancer tissues collected on January 1, 2017 (16). Additionally, the GEO database was searched and GEO datasets (GSE6857, GSE12717, GSE10694, GSE21279, GSE22058, GSE21362, GSE20971, GSE39678, GSE31383, GSE40744, GSE50013, GSE36915, GSE54751, GSE57555, GSE67882, GSE74618, GSE65708 and GSE64989) (17) containing an expression profile or fold-change value of miR-122-5p in HCC and control samples were included. There was no restriction on the specific type of non-cancerous control. Cell line assays or assays without the expression value were excluded. The following information obtained from Genomic Spatial Event (GSE) (https://www.ncbi. nlm.nih.gov/geo/) (18) chips was recorded: Main contributor (the name that ranked first), publishing year, country, sample type, experiment type, the platform of the GSE chips, and the number of patients with $\mathrm{HCC}$ and controls. All expression values for miR-122-5p in GEO were $\log 2$ scaled. The number, mean and standard deviation for the control and experimental groups were calculated based on each single gene chip.
Literature search. Pertinent studies were retrieved in PubMed (https://www.ncbi.nlm.nih.gov/pubmed), Wiley online library (http://onlinelibrary.wiley.com/), Sciencedirect (http://www. sciencedirect.com/), Web of science (http://login.webofknowledge.com/), Cochrane Central Register of Controlled Trials (http://www.cochranelibrary.com/), EMSCO (http://emsco. com/), China National Knowledge Infrastructure (CNKI) (http://www.cnki.net/), China Biology Medicine disc (http://www.sinomed.ac.cn/), Chongqing VIP (http://www. cqvip.com/) and Wan Fang Data (http://www.wanfangdata. com.cn/) using the following key terms: (malignant* OR cancer OR tumor OR tumor OR neoplasm* OR carcinoma) AND (hepatocellular OR liver OR hepatic OR HCC) AND (miR-122 OR miRNA-122 OR microRNA-122 OR miR122 OR miRNA122 OR microRNA122 OR 'miR 122' OR 'miRNA 122' OR 'microRNA 122' OR miR-122-5p OR miRNA-122-5p OR microRNA-122-5p). Studies that did not provide true positive (TP), false positive (FP), true negative (TN) and false negative (FN) results were excluded. The author, publishing year, sample type, number of HCC patients and controls, and the number of TP, FP, TN and FN results were recorded as basic information.

Selection of prospective target genes of miR-122-5p. The potential target mRNAs of miR-122-5p were predicted based on 12 online prediction databases, including miRWalk, TargetScan and miRMap (19). Genes recorded in $>5$ of the 12 prediction databases were selected (20). The selected predicted target genes were further intersected with TCGA and GEO differentially expressed genes. The overlapping genes were considered as potential target genes of miR-122-5p.

Bioinformatic analyses using Gene Ontology (GO), Kyoto Encyclopedia of Genes and Genomes (KEGG), and annotation and the protein-protein interaction (PPI) network. Using the Database for Annotation, Visualization and Integrated Discovery (DAVID; david.abcc.ncifcrf.gov), GO annotation was subsequently conducted based on the prospective target mRNAs. A total of three GO terms, including biological process, cellular component and molecular function, were used to identify the enrichment of target genes. KEGG pathway analysis (www.genome.jp/kegg) was applied to identify noteworthy pathways associated with the selected target genes. The Bingo plug-in component from Cytoscape 3.4.0 (www.cytoscape.org) and GraphPad Prism 7 software (GraphPad Software, Inc., La Jolla, CA, USA) were employed to visualize the network of GO terms and KEGG pathways, respectively (21). GO, KEGG and Protein Analysis Through Evolutionary Relationships (PANTHER) pathway analyses were implemented in DAVID to summarize the essential functions of potential target genes of miR-122-5p (22). To explicitly determine the interactions and connections between hub genes, the PPI network was applied using Search Tool for the Retrieval of Interacting Genes/Proteins (www.string-db.org) (23). The nodes and edges presented the genes and reciprocal actions between genes, whose number would reveal the dominant hub genes of miR-122-5p in HCC. $\mathrm{P}<0.05$ was considered to indicate a statistically significant difference. 

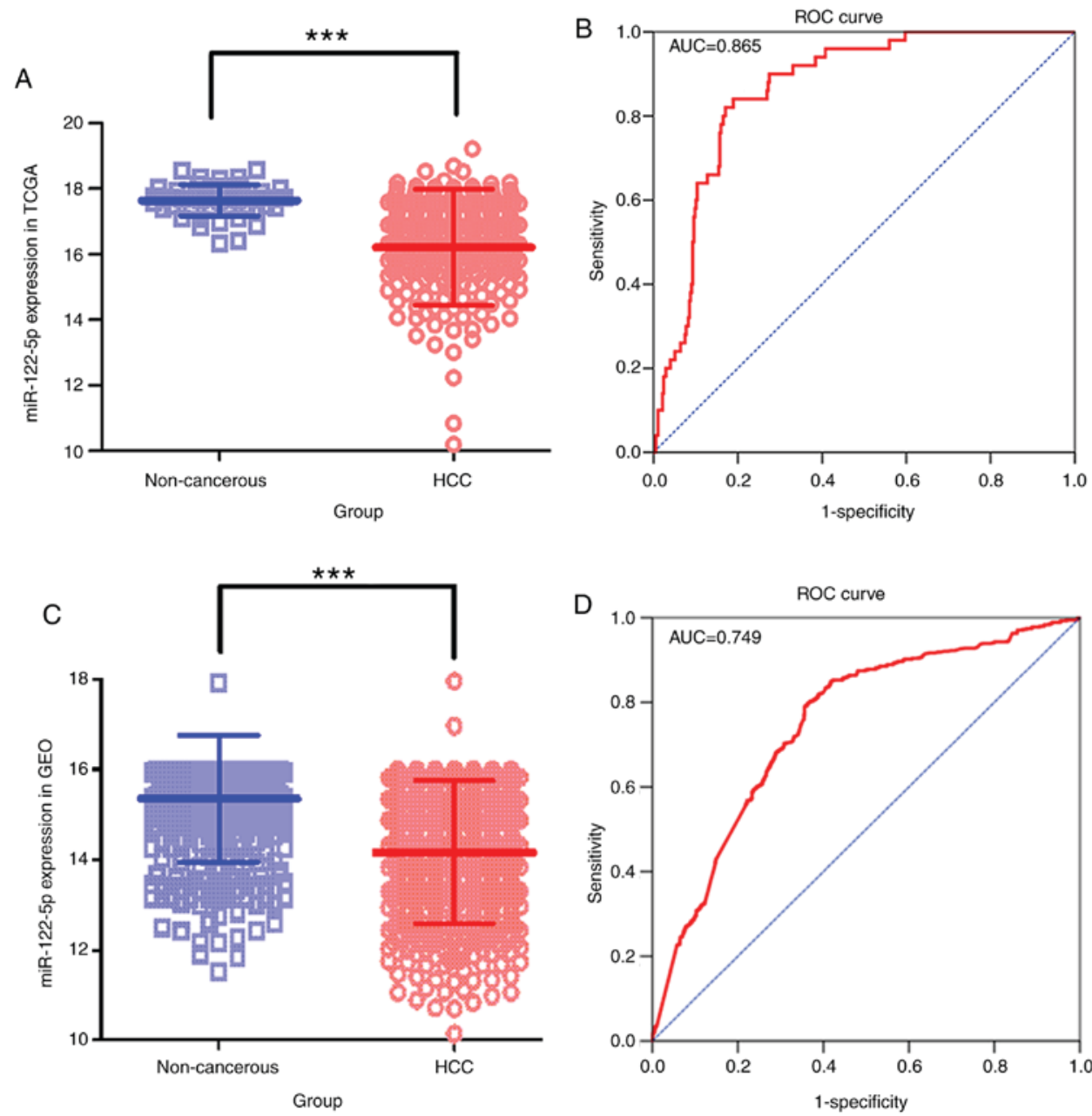

Figure 1. Diagnostic value of miR-122-5p expression in HCC. (A) Scatter chart of groups in TCGA database. (B) ROC curve demonstrating the diagnostic value of miR-122-5p expression based on TCGA database. (C) Scatter chart of groups in the GEO database. (D) ROC curves of miR-122-5p for the diagnosis of HCC based on the GEO database. ${ }^{* * *} \mathrm{P}<0.001$. miR, microRNA; HCC, hepatocellular carcinoma; TCGA, The Cancer Genome Atlas; GEO, Gene Expression Omnibus; ROC, receiver operating characteristic; AUC, area under the curve.

Statistical analysis. Student's t-test for independent samples and one way analysis of variance (with Bonferroni's post-hoc test) was performed using SPSS 19 software (IBM Corp., Armonk, NY, USA) to determine the association between the miR-122-5p expression profile and various clinicopathological parameters. $\mathrm{P}<0.05$ was considered to indicate a statistically significant difference.

SPSS 19 software was applied to generate a receiver operator characteristic (ROC) curve to assess diagnostic efficacy. The relative expression level of miR-122-5p from the microarray included in the GEO was processed separately. All samples were pooled with HCC and controls to conduct another diagnostic trial. Meta-DiSc 1.4 (http://www. hrc.es/investigacion/metadisc_en.htm) was used to generate combined effect and forest plots using a random effects model. The sensitivity (SEN), specificity (SPE), positive likelihood ratio (PLR), negative likelihood ratio (NLR), diagnostic score (DS) and diagnostic odds ratio (DOR) were recorded. The area under the curve (AUC) and point where sensitivity and specificity are equal (index $\mathrm{Q}^{*}$ ) were calculated. Threshold and meta-regression analyses were conducted to determine heterogeneity in the present study, and $\mathrm{P}<0.1$ or $\mathrm{I}^{2}>50 \%$ were considered to represent marked heterogeneity.
The analysis was repeated as aforementioned, and the threshold and meta-regression analyses were performed in Stata14 (StataCorp LLC, College Station, TX, USA). Deek's funnel plot and Fagan plot analyses were performed to examine publication bias and to detect the diagnostic value of miR-122-5p in HCC.

\section{Results}

miR-122-5p expression levels and associated clinicopathological features in TCGA and GEO datasets. The average expression level of miR-122-5p in the TCGA cohort was decreased in the patients with HCC compared with the controls $(\mathrm{P}<0.001$; Fig. 1A). There were 375 patients with $\mathrm{HCC}$ and 50 controls in the TCGA cohort, and 955 patients with HCC and 685 controls in the GEO cohort. From the expression data in 20 GSE chips, all the clinicopathological features mentioned in chips were gathered and associations with miR-122-5p expression levels were investigated. The downregulation of miR-122-5p was associated with HCC tissues $(\mathrm{P}<0.001$; Fig. 1B), tumor vascular invasion $(\mathrm{P}<0.001)$, metastasis $(\mathrm{P}=0.001)$, tissue (compared with serum) $(\mathrm{P}<0.001)$, virus infection state $(\mathrm{P}=0.001)$ and sex $(\mathrm{P}=0.006)$ in the GEO cohort (Table I). 
Table I. Association between the expression of miR-122-5p and clinicopathological parameters in HCC based on the Gene Expression Omnibus database.

miR-122-5p relevant expression

\section{Clinicopathological feature}

Tissue

Non-cancerous

$\mathrm{HCC}$

Age, years

$<50$

$\geq 50$

Sex

Male

Female

Clinical TNM stage

Early stage

Advanced stage

Metastasis

$-$

$+$

Tumor vascular invasion

$\begin{array}{ll}- & 91 \\ + & 81\end{array}$

Alcohol abuse

$-$

$+$

Virus infection state

-

HBV

$\mathrm{HCV}$

Cirrhosis

$$
-
$$$$
+
$$

Chemotherapy

-

$+$

Sample

Tissue

Serum
120

36

685

955

145

117

453

105

82

66

466

171

91

1

0

6

1

5

$$
4
$$$$
42
$$

1,626

14
$15.35 \pm 1.40$

$14.18 \pm 1.58$

$15.40 \pm 1.60$

$15.38 \pm 1.09$

$15.24 \pm 1.54$

$14.78 \pm 1.39$

$15.25 \pm 0.53$

$15.25 \pm 0.44$

$15.36 \pm 0.80$

$15.09 \pm 0.99$

0.001

$14.13 \pm 0.38$

$12.81 \pm 1.09$

$<0.001$

$15.26 \pm 0.48$

0.860

$15.25 \pm 0.49$

$14.55 \pm 1.64$

0.001

$15.47 \pm 1.87$

$14.82 \pm 1.13$

$15.11 \pm 0.82$

0.178

$14.98 \pm 1.07$

$12.35 \pm 0.41$

$12.01 \pm 1.25$

$14.56 \pm 1.51$

$20.10 \pm 2.55$

$<0.001$

0.006

HCC, hepatocellular carcinoma; SD, standard deviation; miR, microRNA; TNM, Tumor-Node-Metastasis; HBV, hepatitis B virus; HCV, hepatitis $\mathrm{C}$ virus.

Diagnostic value based on data from TCGA and GEO. The relative expression level of $\mathrm{miR}-122-5 \mathrm{p}$ data collected from TCGA demonstrated $81.07 \%$ sensitivity and $84.00 \%$ specificity. The area under the ROC curve (AUC) was 0.865 [95\% confidence interval (CI), 0.829-0.896; Fig. 1C]. In the GEO cohort, the overall SEN and the SPE were 66.77 and $79.70 \%$, respectively, with an AUC of 0.749 (95\% CI, 0.726-0.771; Fig. 1D). The diagnostic value of every individual chip was also calculated (Table II) $(15,24-40)$.
Meta-analysis based on the previous literature. In total, 6,854 studies were collected from the literature. Following exclusion of repeated and irrelevant articles, 53 full texts were read. Finally, 4 studies were included (Table III; Fig. 2) (41-44). In the meta-analysis, the pooled SEN, SPE, PLR, NLR, DOR and AUC values were 0.73 (95\% CI, 0.56-0.86), 0.79 (95\% CI, 0.69-0.86), 3.43 (95\% CI, 2.38-4.95), 0.34 (95\% CI, 0.20-0.59), 10.11 (95\% CI, 4.70-21.77) and 0.83 (95\% CI, 0.79-0.86), respectively, when Stata14 was applied (Fig. 3). No significant 


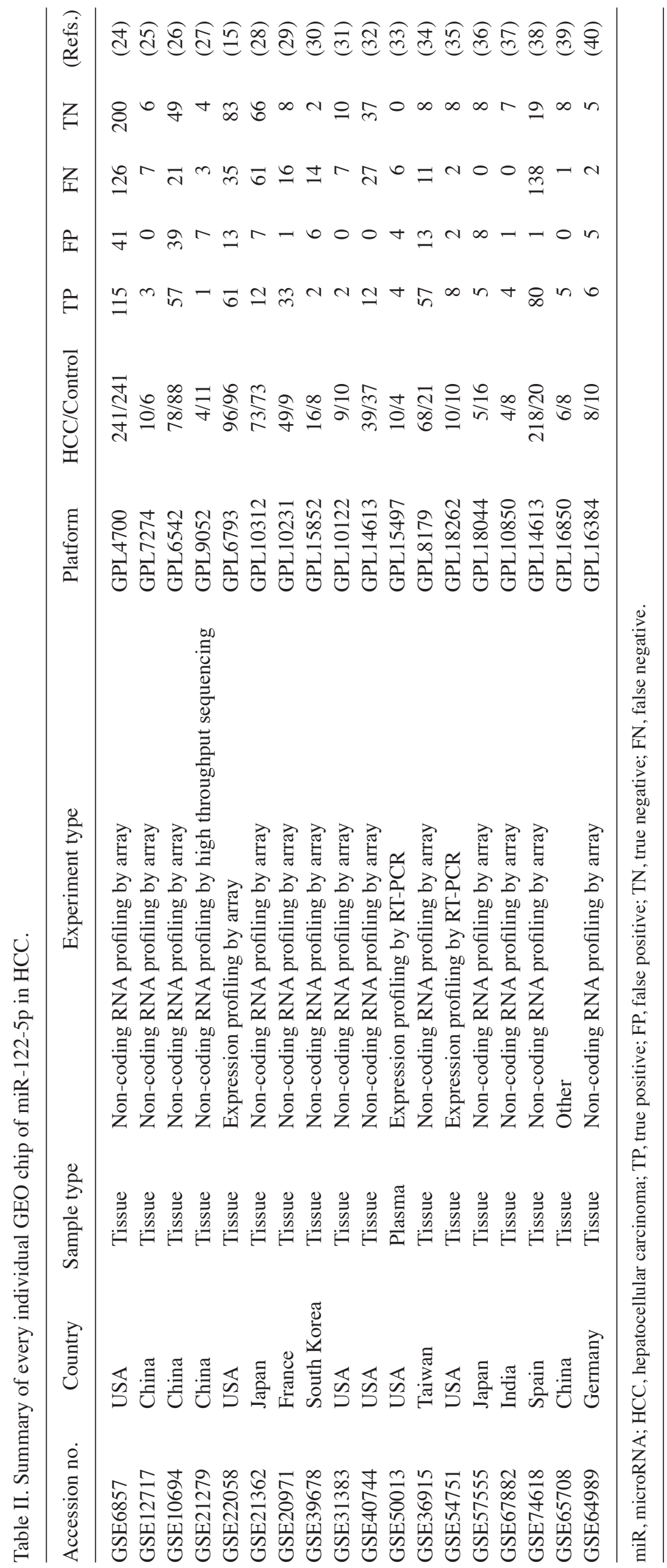


publication bias was observed in the meta-analysis using Deek's funnel plot ( $\mathrm{P}=0.10$; Fig. 4). The pre-test probability value of miR-122-5p in patients with HCC was $20 \%$, and the post-test probability values, considering PLR and NLR results, were 46 and $8 \%$, respectively, based on Fagan's nomogram (Fig. 5). The results were also calculated using Meta-DiSc 1.4 (data not shown).

Overall assessment of diagnostic value. The data from GEO, TCGA and eligible studies were combined in order to perform a pooled diagnostics test. In Stata14, the pooled SEN, SPE, PLR, NLR and DOR values were 0.60 (95\% CI, 0.48-0.71), 0.81 (95\% CI, 0.70-0.89), 3.20 (95\% CI, 1.93-5.30), 0.49 (95\% CI, 0.37-0.65) and 6.49 (95\% CI, 3.23-13.08). The AUC value was 0.76 (95\% CI, 0.72-0.80; Fig. 6). No significant publication bias was observed in the meta-analysis using Deek's funnel plot ( $\mathrm{P}=0.96$; Fig. 7). The pre-test probability value for miR-122-5p expression in patients with HCC was $63 \%$, and the post-test probability values, considering PLR and NLR results, were 84 and 45\%, respectively, based on Fagan's nomogram (Fig. 8). In Meta-DiSc1.4, the pooled SEN, SPE, PLR, NLR and DOR values were also calculated by Meta-DiSc 1.4 (data not shown).

Identification of potential target mRNAs of miR-122-5p. A total of 61,399 target genes were predicted by 12 online software programs, and 5,657 genes that were detected $\geq 5$ times were regarded as potential target genes of miR-122-5p. Additionally, differentially expressed genes assembled from the TCGA and GEO databases were integrated to generate the intersection of the two databases. The 5,657 potential target genes intersected 198 genes, with 3,278 differentially expressed genes from the TCGA database and 5,122 differentially expressed genes from the GEO database. These overlapping genes were used in subsequent bioinformatic analyses.

Bioinformatic analyses of the potential target genes of $m i R-122-5 p$. Further investigations were conducted to determine the functional mechanism of miR-122-5p in the progression of HCC. For the results of GO pathway analysis in DAVID, the potential targets of miR-122-5p were notably associated with the regulation of cell proliferation for cell adhesion $\left(\mathrm{P}=1.22 \times 10^{-4}\right)$, proteinaceous extracellular matrix $\left(\mathrm{P}=1.20 \times 10^{-4}\right)$ and extracellular matrix structural constituent $\left(\mathrm{P}=5.57 \times 10^{-3}\right.$; Table IV; Fig. 9). Furthermore, KEGG pathway analysis identified biosynthesis of amino acids, carbon metabolism, biosynthesis of antibiotics, arginine and proline metabolism, arginine biosynthesis, Chagas disease (American trypanosomiasis), amoebiasis, glycolysis/gluconeogenesis, the pentose phosphate pathway and toxoplasmosis as significant $(\mathrm{P}<0.05)$. In addition, the most enriched term in the PANTHER analysis was salvage pathways of pyrimidine deoxyribonucleotides (Table V; Fig. 10). In the PPI analysis, the network demonstrated 198 nodes and 57 edges (Fig. 11A). Genes with a combined score $>0.900$, based on available experimental data and importing known protein complexes from curated databases (23), including minichromosome maintenance complex component (MCM)4, MCM8, MCM10, chromatin licensing and DNA replication factor 1, cell division cycle (CDC) 6, CDC25A and checkpoint kinase 1, were

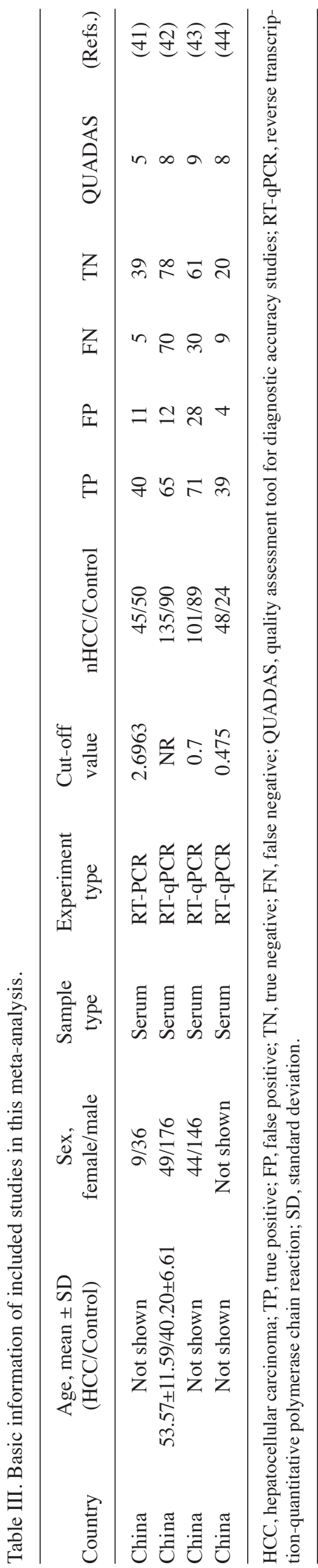




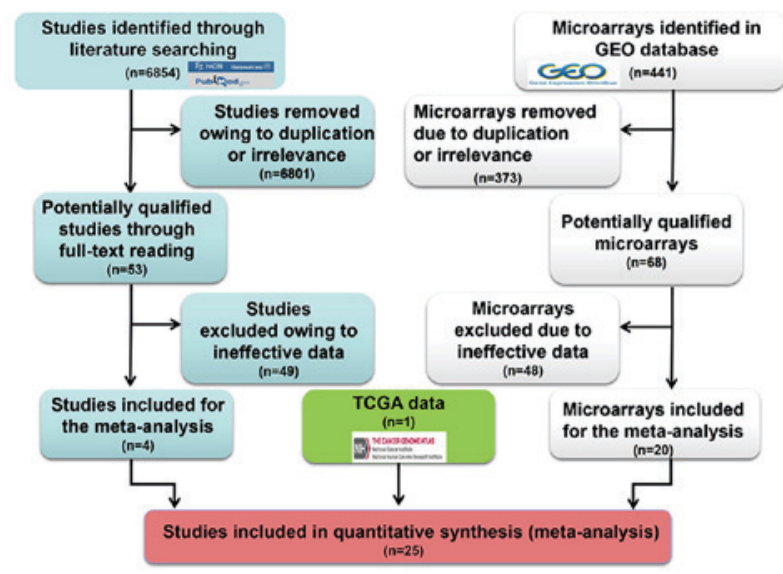

Figure 2. Flow chart of the study selection in the meta-analysis. TCGA, The Cancer Genome Atlas; GEO, Gene Expression Omnibus.

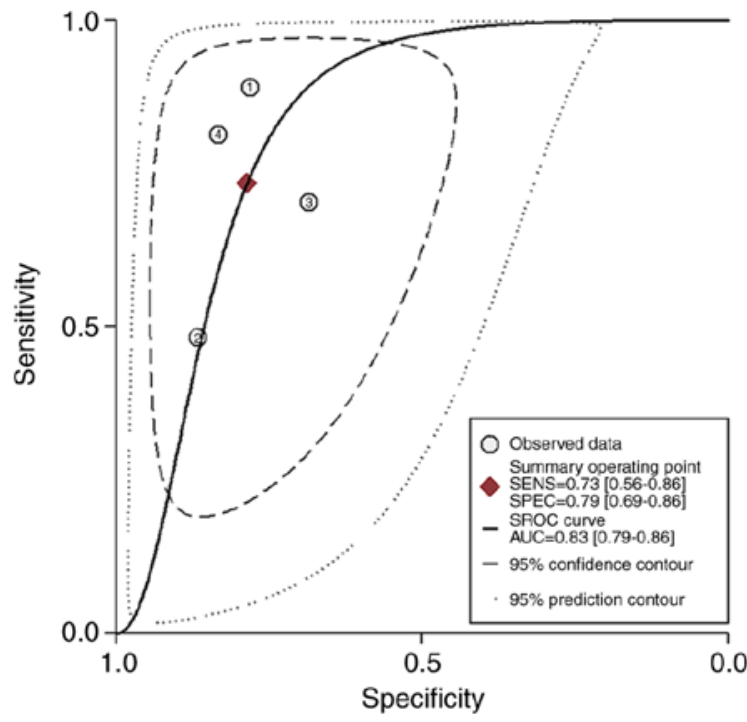

Figure 3. The SROC curve analysis for miR-122-5p in the 4 included studies by Stata14. SROC curves summarize the overall diagnostic accuracy. SROC summary receiver operating characteristic; SENS, sensitivity; SPEC, specificity; AUC, area under the curve.

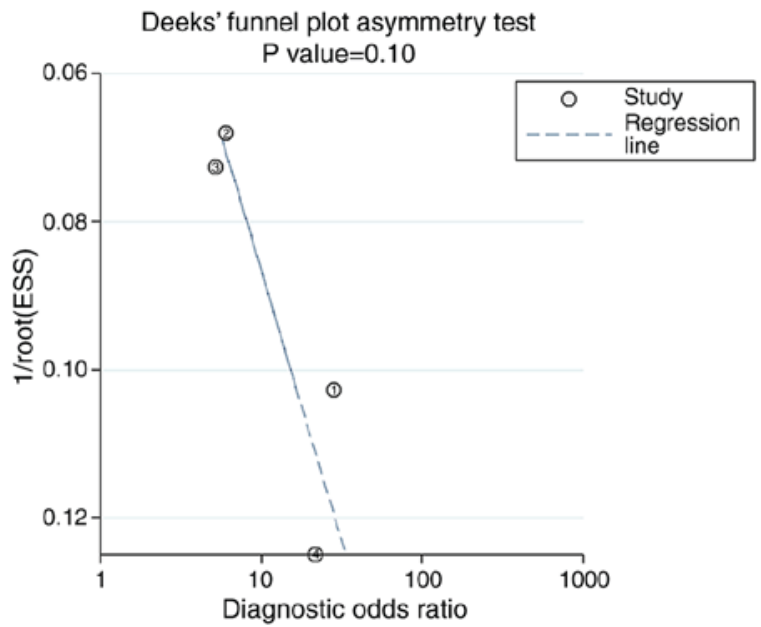

Figure 4. Deek's funnel plot evaluating the publication bias based on the included studies in Stata14. $\mathrm{P}=0.10$, no significant publication bias was observed in the meta-analysis. ESS, effective sample size.

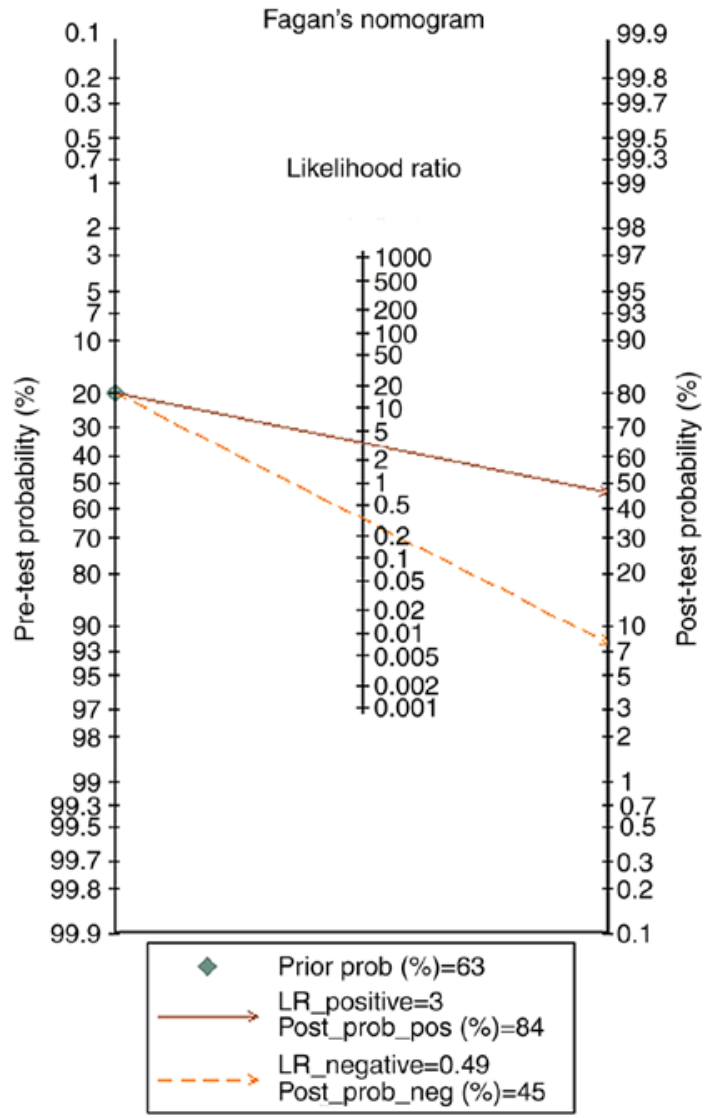

Figure 5. Fagan's nomogram detecting the clinical use of miR-122-5p in the qualified studies in HCC based on Stata14. The pre-test probability value of miR-122-5p in HCC was $20 \%$, and the post-test probability values, considering the LR_positive and LR_negative results, were 46 and $8 \%$, respectively. miR, microRNA; HCC, hepatocellular carcinoma; LR_positive, positive likelihood ratio; LR_negative, negative likelihood ratio; post_prob_pos, post-test positive probability; post_prob_neg, post-test negative probability.

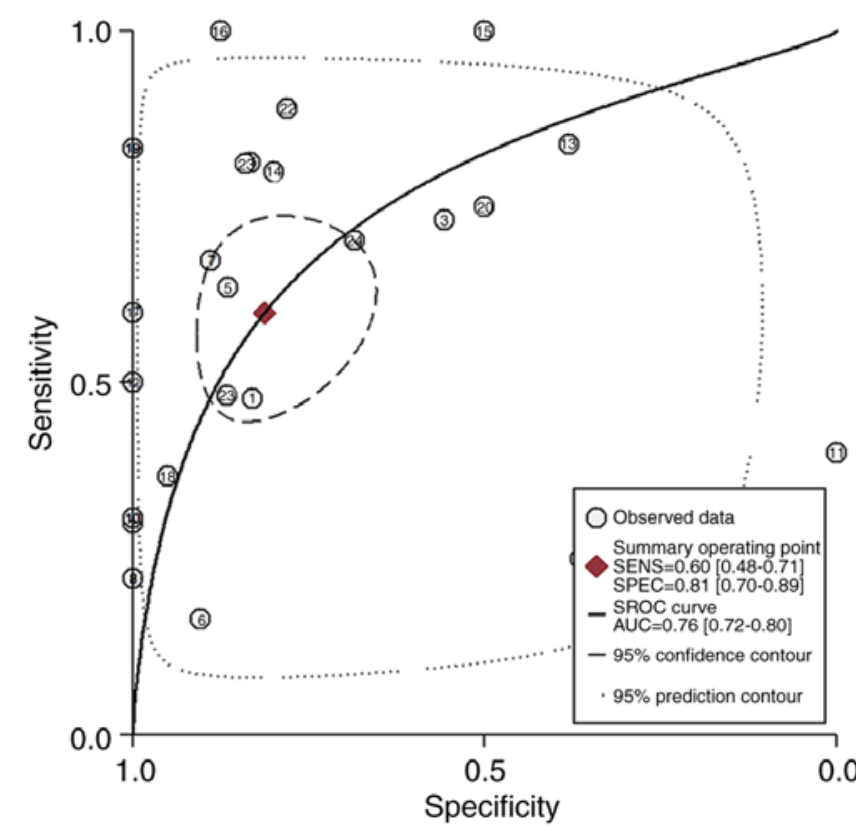

Figure 6. The SROC curve analysis for miR-122-5p based on The Cancer Genome Atlas, Gene Expression Omnibus and eligible studies in Stata14. SROC curves summarize the overall diagnostic accuracy. SROC, summary receiver operating characteristic; SENS, sensitivity; SPEC, specificity; AUC, area under the curve. 
Deeks' funnel plot asymmetry test pvalue $=0.96$

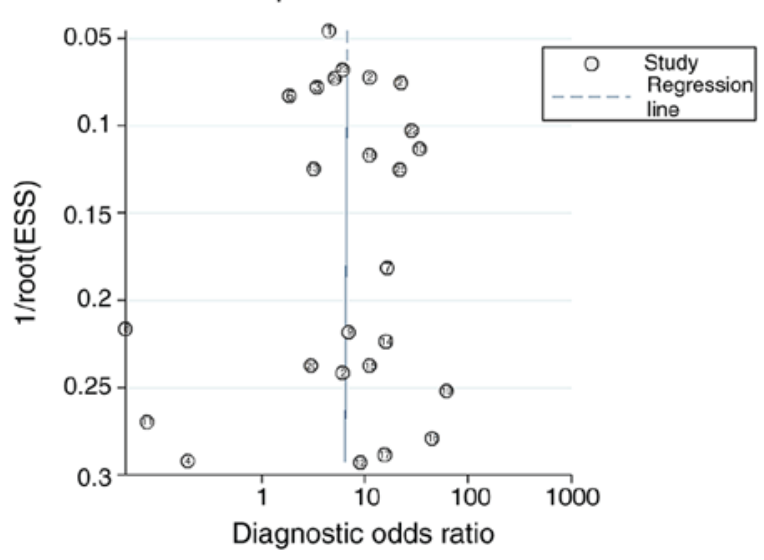

Figure 7. Deek's funnel plot examining the publication bias based on The Cancer Genome Atlas, Gene Expression Omnibus and eligible studies in Stata14. No significant publication bias was observed in the meta-analysis; $\mathrm{P}=0.96$. ESS, effective sample size.

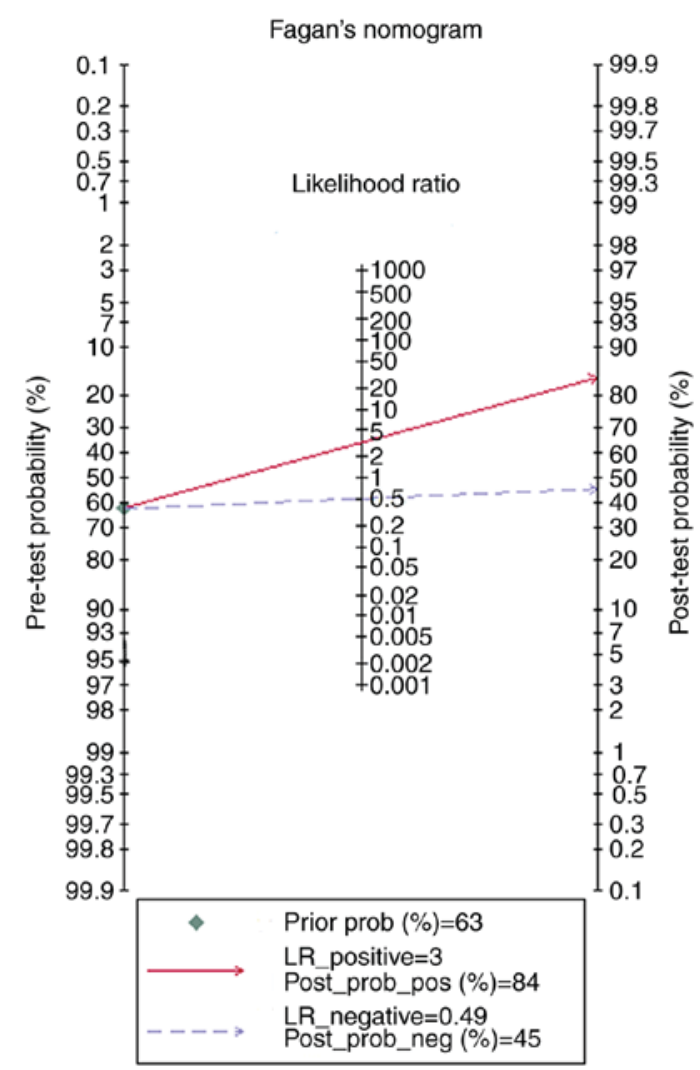

Figure 8. The Fagan plot was displayed to test the diagnostic value of miR-122-5p based on The Cancer Genome Atlas, Gene Expression Omnibus and eligible studies in HCC by Stata14. The pre-test probability value of miR-122-5p in HCC was 63\%, and the post-test probability values, considering the LR_positive and LR_negative results, were 84 and $45 \%$, respectively. miR, microRNA; HCC, hepatocellular carcinoma; LR_positive, positive likelihood ratio; LR_negative, negative likelihood ratio; post_prob_pos, post-test positive probability; post_prob_neg, post-test negative probability.

defined as hub genes. CDC6, MCM4 and MCM8, with 24, 24 and 22 interactions with other hub genes in the network, respectively, were recognized as hub genes of the highest significance (Fig. 11B).

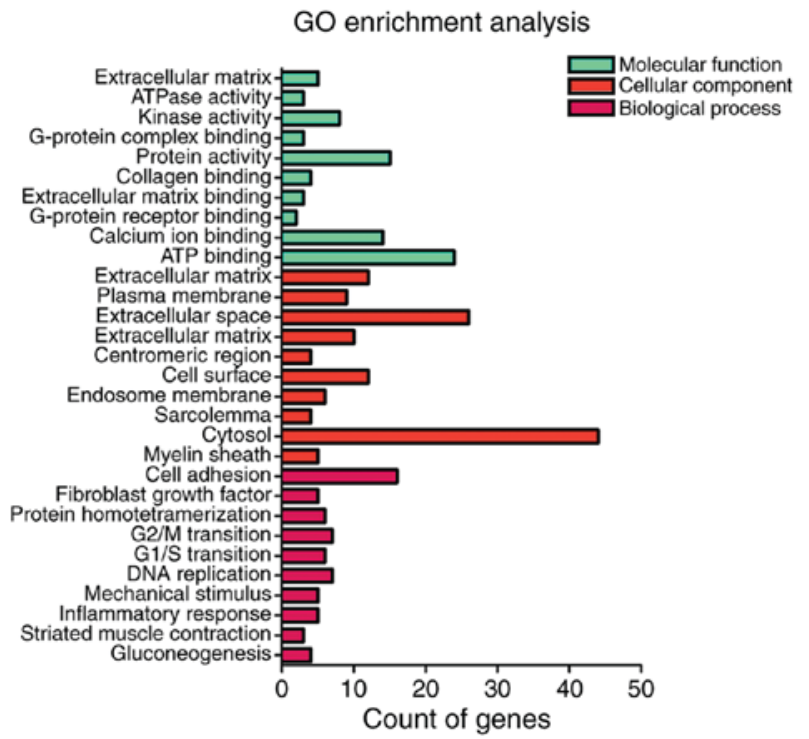

Figure 9. GO functional annotation according to the putative target genes of miR-122-5p in HCC. The top 10 terms with highest significance in every section of the GO analysis are presented respectively with the number of enriched target genes. GO, gene ontology; miR, microRNA; HCC, hepatocellular carcinoma.

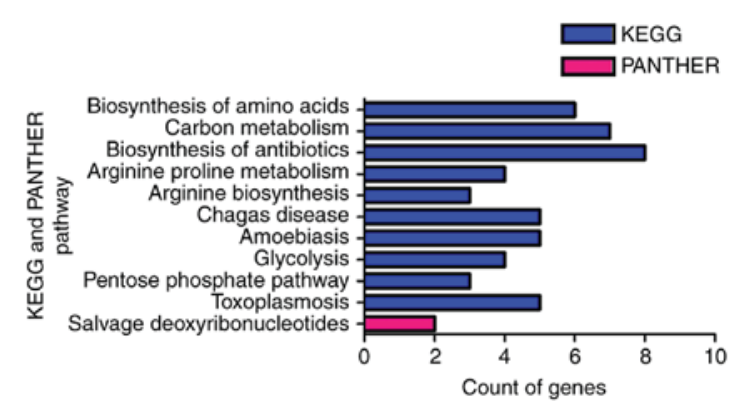

Figure 10. KEGG and PANTHER pathway enrichment analysis of miR-122-5p target genes. The number of genes is indicated in the figure. KEGG, Kyoto Encyclopedia of Genes and Genomes; PANTHER, Protein Analysis Through Evolutionary Relationships; miR, microRNA.

\section{Discussion}

A number of studies have confirmed that the downregulation of miR-122-5p in HCC was associated with metastasis and a poor prognosis $(45,46)$. Therefore, the present study further investigated these conclusions. The expression of miR-122-5p was decreased in patients with HCC compared with that in controls in the TCGA and GEO databases. Additionally, decreased miR-122-5p expression was significantly associated with metastasis and vascular invasion in the GEO dataset, suggesting that the downregulation of miR-122-5p may lead to a poor prognosis, and that miR-122-5p may act as a tumor suppressor in HCC. Furthermore, miR-122-5p expression varied in different virus infection states, indicating that miR-122-5p may be associated with virus-associated HCC. In tissues, miR-122-5p expression was decreased compared with that in serum. However, reflecting the limited number of serum samples, further studies are required to confirm distinctions in the diagnostic accuracy between tissues and serum. 
Table IV. The top 10 most enriched terms in every GO section, based on the target genes of miR-122-5p in hepatocellular carcinoma.

\begin{tabular}{|c|c|c|c|}
\hline Category & Term & Count & P-value \\
\hline GOTERM_BP & GO:0007155 cell adhesion & 16 & $1.22 \times 10^{-4}$ \\
\hline GOTERM_BP & GO:0044344 cellular response to fibroblast growth factor stimulus & 5 & $2.76 \times 10^{-4}$ \\
\hline GOTERM_BP & GO:0051289 protein homotetramerization & 6 & $4.42 \times 10^{-4}$ \\
\hline GOTERM_BP & GO:0000086 G2/M transition of mitotic cell cycle & 7 & $3.51 \times 10^{-3}$ \\
\hline GOTERM_BP & GO:0000082 G1/S transition of mitotic cell cycle & 6 & $4.71 \times 10^{-3}$ \\
\hline GOTERM_BP & GO:0006260 DNA replication & 7 & $6.38 \times 10^{-3}$ \\
\hline GOTERM_BP & GO:0071260 cellular response to mechanical stimulus & 5 & $6.96 \times 10^{-3}$ \\
\hline GOTERM_BP & GO:0050729 positive regulation of inflammatory response & 5 & $7.67 \times 10^{-3}$ \\
\hline GOTERM_BP & GO:0006941 striated muscle contraction & 3 & $8.16 \times 10^{-3}$ \\
\hline GOTERM_BP & GO:0006094 gluconeogenesis & 4 & $1.14 \times 10^{-2}$ \\
\hline GOTERM_CC & GO:0005578 proteinaceous extracellular matrix & 12 & $1.20 \times 10^{-4}$ \\
\hline GOTERM_CC & GO:0009897 external side of plasma membrane & 9 & $1.80 \times 10^{-3}$ \\
\hline GOTERM_CC & GO:0005615 extracellular space & 26 & $3.52 \times 10^{-3}$ \\
\hline GOTERM_CC & GO:0031012 extracellular matrix & 10 & $3.98 \times 10^{-3}$ \\
\hline GOTERM_CC & GO:0000775 chromosome, centromeric region & 4 & $2.16 \times 10^{-2}$ \\
\hline GOTERM_CC & GO:0009986 cell surface & 12 & $2.70 \times 10^{-2}$ \\
\hline GOTERM_CC & GO:0010008 endosome membrane & 6 & $4.47 \times 10^{-2}$ \\
\hline GOTERM_CC & GO:0042383 sarcolemma & 4 & $5.92 \times 10^{-2}$ \\
\hline GOTERM_CC & GO:0005829 cytosol & 44 & $6.95 \times 10^{-2}$ \\
\hline GOTERM_CC & GO:0043209 myelin sheath & 5 & $7.45 \times 10^{-2}$ \\
\hline GOTERM_MF & GO:0005201 extracellular matrix structural constituent & 5 & $5.57 \times 10^{-3}$ \\
\hline GOTERM_MF & GO:0043225 anion transmembrane-transporting ATPase activity & 3 & $5.77 \times 10^{-3}$ \\
\hline GOTERM_MF & GO:0016301 kinase activity & 8 & $1.45 \times 10^{-2}$ \\
\hline GOTERM_MF & GO:0031683 G-protein beta/gamma-subunit complex binding & 3 & $1.87 \times 10^{-2}$ \\
\hline GOTERM_MF & GO:0042803 protein homodimerization activity & 15 & $2.36 \times 10^{-2}$ \\
\hline GOTERM_MF & GO:0005518 collagen binding & 4 & $2.58 \times 10^{-2}$ \\
\hline GOTERM_MF & GO:0050840 extracellular matrix binding & 3 & $3.08 \times 10^{-2}$ \\
\hline GOTERM_MF & GO:0031821 G-protein coupled serotonin receptor binding & 2 & $4.17 \times 10^{-2}$ \\
\hline GOTERM_MF & GO:0005509 calcium ion binding & 14 & $4.20 \times 10^{-2}$ \\
\hline GOTERM_MF & GO:0005524 ATP binding & 24 & $4.48 \times 10^{-2}$ \\
\hline
\end{tabular}

GO, Gene Ontology; miR, microRNA; BP, biological process; CC, cellular component; MF, molecular function.

Table V. KEGG and PANTHER Pathway analysis of potential target genes of miR-122-5p.

\begin{tabular}{|c|c|c|c|}
\hline Category & Term & Count & P-value \\
\hline KEGG_PATHWAY & hsa01230:Biosynthesis of amino acids & 6 & $1.67 \times 10^{-3}$ \\
\hline KEGG_PATHWAY & hsa01200:Carbon metabolism & 7 & $2.00 \times 10^{-3}$ \\
\hline KEGG_PATHWAY & hsa01130:Biosynthesis of antibiotics & 8 & $1.18 \times 10^{-2}$ \\
\hline KEGG_PATHWAY & hsa00330:Arginine and proline metabolism & 4 & $2.05 \times 10^{-2}$ \\
\hline KEGG_PATHWAY & hsa00220:Arginine biosynthesis & 3 & $2.25 \times 10^{-2}$ \\
\hline KEGG_PATHWAY & hsa05142:Chagas disease (American trypanosomiasis) & 5 & $3.33 \times 10^{-2}$ \\
\hline KEGG_PATHWAY & hsa05146:Amoebiasis & 5 & $3.54 \times 10^{-2}$ \\
\hline KEGG_PATHWAY & hsa00010:Glycolysis/Gluconeogenesis & 4 & $4.35 \times 10^{-2}$ \\
\hline KEGG_PATHWAY & hsa00030:Pentose phosphate pathway & 3 & $4.49 \times 10^{-2}$ \\
\hline KEGG_PATHWAY & hsa05145:Toxoplasmosis & 5 & $4.93 \times 10^{-2}$ \\
\hline PANTHER_PATHWAY & P02774:Salvage pyrimidine deoxyribonucleotides & 2 & $5.89 \times 10^{-2}$ \\
\hline
\end{tabular}

KEGG, Kyoto Encyclopedia of Genes and Genomes; PANTHER, Protein Analysis Through Evolutionary Relationships; miR, microRNA. 

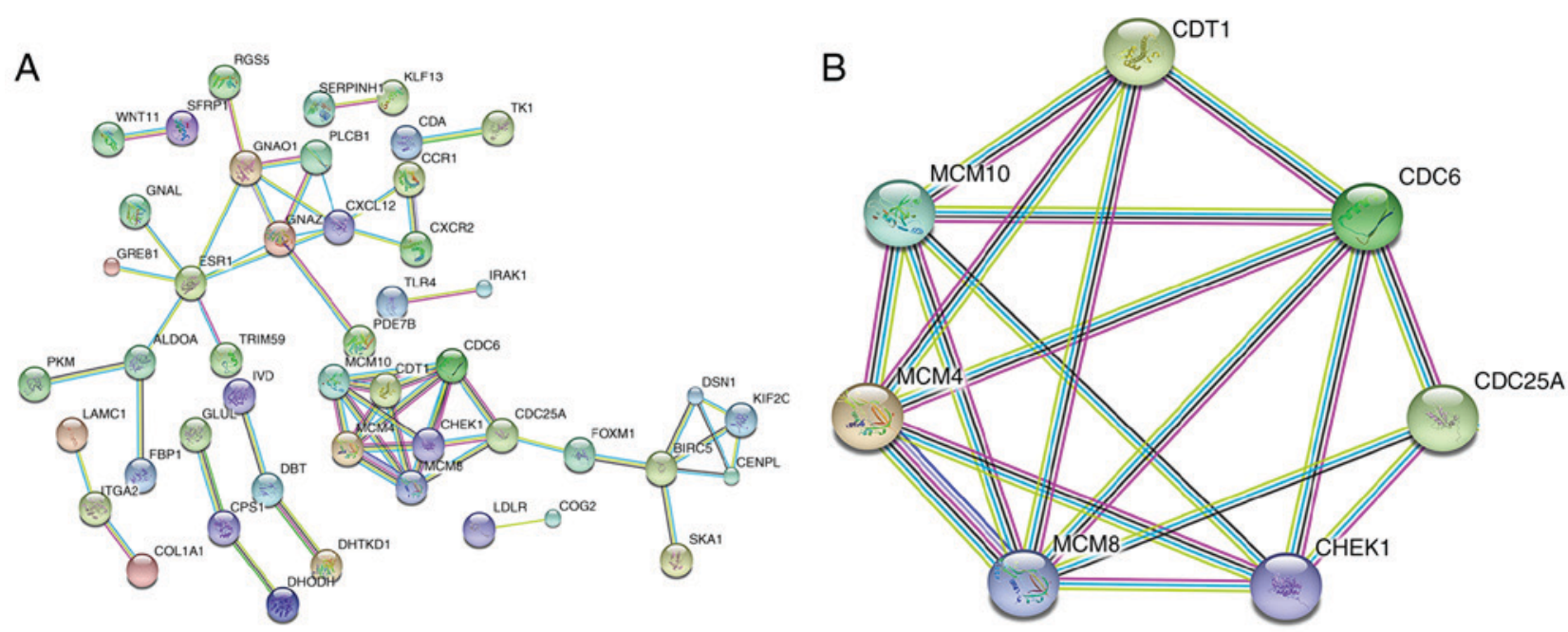

Figure 11. The PPI network of potential target genes of miR-122-5p in HCC. (A) The network comprising 198 potential target genes of miR-122-5p. The PPI enrichment P-value was $1.13 \times 10^{-11}$ for the network containing 198 nodes and 57 edges. The disconnected nodes are not presented. (B) The 7 hub genes with the most interaction lines in the PPI analysis. PPI, protein-protein interaction; miR, microRNA; HCC, hepatocellular carcinoma.

With regards to diagnostic value, the pooled AUC was 0.76 (95\% CI, 0.72-0.80), suggesting that miR-122-5p possessed a moderate degree of accuracy in diagnostic tests and that it may be a promising biomarker for the diagnosis of patients with HCC. A discrepancy in sample type and patients may reflect the observed differences in the AUC among TCGA, GEO and meta-analysis studies. Li et al (47), He et al (48) and Huang et al (49) conducted integrated research to examine the diagnostic value of miR-122-5p, and the AUC values were $0.81,0.78$ and 0.77 , respectively, consistent with the results of the present study. In search strategy, Wu et al (46) applied 2 public databases: PubMed and Embase, Qi et al (44) applied Medline and CancerLit Embase prior to July 31, 2015. Huang et al (49) analyzed the diagnostic value of miRNAs in patients with HCC utilizing the Medline, Embase and CNKI databases. The present study aimed to explore the potential clinical value of miR-122-5p in HCC. In addition to pertinent literature regarding miR-122-5p, data from TCGA and GEO public databases were also extracted. The SEN, SPE, PLR, NLR, DS and DOR values were determined in Meta-DiSc 1.4. The aforementioned study was repeated, excluding threshold analysis and meta-regression in Stata14. Deek's funnel plot and Fagan plot were displayed to test the publication bias and to detect the diagnostic value of miR-122-5p in HCC. The heterogeneity in the meta-analysis of these studies did not reflect a threshold effect or clinical features. Subgroup and meta-regression analyses were not performed, reflecting the limited number of studies collected. The source of heterogeneity in the pooled meta-analysis was not determined by meta-regression.

In the PPI network, CDC6, MCM4 and MCM8 were highlighted as the most significant hub genes with multiple interactions in the network. Further examination of these notable genes would reveal the function for miR-122-5p in HCC. CDC6 is a necessary regulator of DNA replication and is inextricably associated with tumorigenesis (50). High CDC6 expression has been associated with various types of cancer. In ovarian cancer, Deng et al (51) discovered that the elevated expression of CDC6 may accelerate cell proliferation and worsen prognosis. In addition, CDC6 may participate in chemotherapy resistance in bladder cancer (52). Previous studies have also demonstrated oncogenetic functions for CDC6 in HCC $(53,54)$. Xiong et al $(55)$ demonstrated that CDC6 overexpression may increase susceptibility to HCC, and the Cdc6-515A $>$ G polymorphism may attenuate CDC6 expression to decrease the risk of carcinogenesis. Therefore, studies focused on approaches to downregulate CDC6 have been reported. HKH40A was utilized to disrupt the cell cycle and to promote apoptosis, during which CDC6 was downregulated (56). MCM4 is also an essential replication modulator. In cervical cancer (57), non-small cell lung cancer (58) and laryngeal squamous cell carcinoma (59), the upregulation of MCM4 was also observed. Studach et al (60) observed upregulation of MCM4 in HCC in X/c-myc bitransgenics, indicating the identical expression profile of MCM4 in HCC. However, the MCM4 polymorphism may generate contradictory results. For example, Nan et al (61) reported that MCM4 may decrease the risk of HCC. Another notable hub gene of miR-122-5p, MCM8, which is also a gene replication regulator, may modulate DNA replication through interactions with other MCM proteins, including the aforementioned MCM4 (62). MCM8 was overexpressed in chronic myelogenous leukemia (63) and the overexpression of MCM8 was associated with the poor prognosis in pancreatic cancer (64). However, to the best of our knowledge, there are no available studies regarding the function of MCM8 in HCC. As the hub genes of miR-122-5p with the most interactions with other hub genes, CDC6, MCM4 and MCM8 all exerted the function of genome replication. Consistently, regulation of cell proliferation for cell adhesion was also highlighted in the $\mathrm{GO}$ analysis. Therefore, we hypothesized that miR-122-5p may be involved in the biological process of gene replication. Considering that miR-122-5p exhibited significantly decreased expression in HCC, whereas CDC6 and MCM4 were upregulated, miR-122-5p may represent a therapeutic target and biomarker for HCC. 
In conclusion, the downregulation of miR-122-5p in HCC demonstrated diagnostic value worthy of further attention. Furthermore, this molecule may function as a tumor suppressor by modulating genome replication. Additional experiments and studies are required to verify this discovery.

\section{Acknowledgements}

The present study was partly supported by the Fund of National Natural Science Foundation of China (grant nos. NSFC81260222 and NSFC81060202), the Fund of Youth Science Foundation of Guangxi Medical University (grant no. GXMUYSF201624) and the Guangxi Medical University Students Innovative Project (grant no. WLXSZX17001).

\section{Competing interests}

The authors declare that they have no competing interests.

\section{References}

1. El-Serag HB: Hepatocellular carcinoma. N Engl J Med 365: 1118-1127, 2011.

2. Ferenci P, Fried M, Labrecque D, Bruix J, Sherman M, Omata M, Heathcote J, Piratsivuth T, Kew M, Otegbayo JA, et al: Hepatocellular carcinoma (HCC): A global perspective. J Clin Gastroenterol 44: 239-245, 2010

3. Oliveri RS, Wetterslev J and Gluud C: Transarterial (chemo) embolisation for unresectable hepatocellular carcinoma. Cochrane Database Syst Rev 3: CD004787, 2011.

4. Feng M and Ben-Josef E: Radiation therapy for hepatocellular carcinoma. Semin Radiat Oncol 21: 271-277, 2011.

5. Marrero JA, Feng Z, Wang Y, Nguyen MH, Befeler AS, Roberts LR, Reddy KR, Harnois D, Llovet JM, Normolle D, et al: Alpha-fetoprotein, des-gamma carboxyprothrombin, and lectin-bound alpha-fetoprotein in early hepatocellular carcinoma. Gastroenterology 137: 110-118, 2009.

6. Baig JA, Alam JM, Mahmood SR, Baig M, Shaheen R, Sultana I and Waheed A: Hepatocellular carcinoma (HCC) and diagnostic significance of A-fetoprotein (AFP). J Ayub Med Coll Abbottabad 21: 72-75, 2009.

7. Collier J and Sherman M: Screening for hepatocellular carcinoma. Hepatology 27: 273-278, 1998.

8. Shaker O, Alhelf M, Morcos G and Elsharkawy A: miRNA-101-1 and miRNA-221 expressions and their polymorphisms as biomarkers for early diagnosis of hepatocellular carcinoma. Infect Genet Evol 51: 173-181, 2017.

9. Ye W, Li J, Fang G, Cai X, Zhang Y, Zhou C, Chen L and Yang W: Expression of microRNA 638 and sex-determining region Y-box 2 in hepatocellular carcinoma: Association between clinicopathological features and prognosis. Oncol Lett 15: 7255-7264, 2018

10. Li F, Wang F, Zhu C, Wei Q, Zhang T and Zhou YL: miR-221 suppression through nanoparticle-based miRNA delivery system for hepatocellular carcinoma therapy and its diagnosis as a potential biomarker. Int J Nanomed 13: 2295-2307, 2018.

11. Bartel DP: MicroRNAs: Genomics, biogenesis, mechanism, and function. Cell 116: 281-297, 2004.

12. Ambros V: The functions of animal microRNAs. Nature 431: 350-355, 2004

13. Hua HW, Jiang F, Huang Q, Liao Z and Ding G: MicroRNA-153 promotes Wnt/ $\beta$-catenin activation in hepatocellular carcinoma through suppression of WWOX. Oncotarget 6: 3840-3847, 2015.

14. Wu LM, Ji JS, Yang Z, Xing CY, Pan TT, Xie HY, Zhang F, Zhuang L, Zhou L and Zheng SS: Oncogenic role of microRNA-423-5p in hepatocellular carcinoma. Hepatobiliary Pancreat Dis Int 14: 613-618, 2015.

15. Burchard J, Zhang C, Liu AM, Poon RT, Lee NP, Wong KF, Sham PC,Lam BY,Ferguson MD, Tokiwa G, et al: microRNA-122 as a regulator of mitochondrial metabolic gene network in hepatocellular carcinoma. Mol Syst Biol 6: 402, 2010.

16. Hutter $\mathrm{C}$ and Zenklusen JC: The cancer genome atlas: Creating lasting value beyond its data. Cell 173: 283-285, 2018.
17. Barrett T, Wilhite SE, Ledoux P, Evangelista C, Kim IF, Tomashevsky M, Marshall KA, Phillippy KH, Sherman PM, Holko M, et al: NCBI GEO: Archive for functional genomics data sets-update. Nucleic Acids Res 41: D991-D995, 2013.

18. Danford T, Rolfe A and Gifford D: GSE: A comprehensive database system for the representation, retrieval, and analysis of microarray data. Pac Symp Biocomput: 539-550, 2008.

19. Lee $M$ and Lee H: DMirNet: Inferring direct microRNA-mRNA association networks. BMC Syst Biol 10: 125, 2016.

20. Huang S, Xie Y, Yang P, Chen P and Zhang L: HCV core protein-induced down-regulation of microRNA-152 promoted aberrant proliferation by regulating Wnt1 in HepG2 cells. PLoS One 9: e81730, 2014

21. Shannon P, Markiel A, Ozier O, Baliga NS, Wang JT, Ramage D, Amin N, Schwikowski B and Ideker T: Cytoscape: A software environment for integrated models of biomolecular interaction networks. Genome Res 13: 2498-2504, 2003.

22. Jiao X, Sherman BT, Huang da W, Stephens R, Baseler MW, Lane HC and Lempicki RA: DAVID-WS: A stateful web service to facilitate gene/protein list analysis. Bioinformatics 28 : 1805-1806, 2012

23. Szklarczyk D, Morris JH, Cook H, Kuhn M, Wyder S, Simonovic M, Santos A, Doncheva NT, Roth A, Bork P, et al: The STRING database in 2017: Quality-controlled protein-protein association networks, made broadly accessible. Nucleic Acids Res 45: D362-D368, 2017.

24. Budhu A, Jia HL, Forgues M, Liu CG, Goldstein D, Lam A, Zanetti KA, Ye QH, Qin LX, Croce CM, et al: Identification of metastasis-related microRNAs in hepatocellular carcinoma. Hepatology 47: 897-907, 2008.

25. Su H, Yang JR, Xu T, Huang J, Xu L, Yuan Y and Zhuang SM: MicroRNA-101, down-regulated in hepatocellular carcinoma, promotes apoptosis and suppresses tumorigenicity. Cancer Res 69: 1135-1142, 2009.

26. Li W, Xie L, He X, Li J, Tu K, Wei L, Wu J, Guo Y, Ma X, Zhang $\mathrm{P}$, et al: Diagnostic and prognostic implications of microRNAs in human hepatocellular carcinoma. Int $\mathrm{J}$ Cancer 123: 1616-1622, 2008

27. Hou J, Lin L, Zhou W, Wang Z, Ding G, Dong Q, Qin L, Wu X, Zheng Y, Yang Y, et al: Identification of miRNomes in human liver and hepatocellular carcinoma reveals miR-199a/b-3p as therapeutic target for hepatocellular carcinoma. Cancer Cell 19: 232-243, 2011.

28. Sato F, Hatano E, Kitamura K, Myomoto A, Fujiwara T, Takizawa S, Tsuchiya S, Tsujimoto G, Uemoto S and Shimizu K: MicroRNA profile predicts recurrence after resection in patients with hepatocellular carcinoma within the Milan Criteria. PLoS One 6: e16435, 2011.

29. Cairo S, Wang Y, de Reyniès A, Duroure K, Dahan J, Redon MJ, Fabre M, McClelland M, Wang XW, Croce CM and Buendia MA: Stem cell-like micro-RNA signature driven by Myc in aggressive liver cancer. Proc Natl Acad Sci USA 107: 20471-20476, 2010.

30. Noh JH, Chang YG, Kim MG, Jung KH, Kim JK, Bae HJ, Eun JW, Shen Q, Kim SJ, Kwon SH, et al: MiR-145 functions as a tumor suppressor by directly targeting histone deacetylase 2 in liver cancer. Cancer Lett 335: 455-462, 2013.

31. Han K, Li J, Zhao H, Liang P, Huang X, Zheng L, Li Y, Yang T and Wang L: Identification of the typical miRNAs and target genes in hepatocellular carcinoma. Mol Med Rep 10: 229-235, 2014.

32. Diaz G, Melis M, Tice A, Kleiner DE, Mishra L, Zamboni F and Farci P: Identification of microRNAs specifically expressed in hepatitis $\mathrm{C}$ virus-associated hepatocellular carcinoma. Int $\mathrm{J}$ Cancer 133: 816-824, 2013.

33. Shen J, Wang A, Wang Q, Gurvich I, Siegel AB, Remotti H and Santella RM: Exploration of genome-wide circulating microRNA in hepatocellular carcinoma: MiR-483-5p as a potential biomarker. Cancer Epidemiol Biomarkers Prev 22: 2364-2373, 2013.

34. Huang DH, Wang GY, Zhang JW, Li Y, Zeng XC and Jiang N: miR-501-5p regulates CYLD expression and promotes cell proliferation in human hepatocellular carcinoma. Jpn J Clin Oncol 45: 738-744, 2015.

35. Zhao L and Zhang Y: miR-342-3p affects hepatocellular carcinoma cell proliferation via regulating NF- $\kappa$ B pathway. Biochem Biophys Res Commun 457: 370-377, 2015.

36. Murakami Y, Kubo S, Tamori A, Itami S, Kawamura E, Iwaisako K, Ikeda K, Kawada N, Ochiya T and Taguchi YH: Comprehensive analysis of transcriptome and metabolome analysis in intrahepatic cholangiocarcinoma and hepatocellular carcinoma. Sci Rep 5: 16294, 2015. 
37. Ding W, Yang H, Gong S, Shi W, Xiao J, Gu J, Wang Y and $\mathrm{He} \mathrm{B}$ : Candidate miRNAs and pathogenesis investigation for hepatocellular carcinoma based on bioinformatics analysis. Oncol Lett 13: 3409-3414, 2017.

38. Martinez-Quetglas I, Pinyol R, Dauch D, Torrecilla S, Tovar V, Moeini A, Alsinet C, Portela A, Rodriguez-Carunchio L, Solé M, et al: IGF2 Is up-regulated by epigenetic mechanisms in hepatocellular carcinomas and is an actionable oncogene product in experimental models. Gastroenterology 151: 1192-1205, 2016.

39. Lin XJ, Chong Y, Guo ZW, Xie C, Yang XJ, Zhang Q, Li SP, Xiong Y, Yuan Y, Min J, et al: A serum microRNA classifier for early detection of hepatocellular carcinoma: A multicentre, retrospective, longitudinal biomarker identification study with a nested case-control study. Lancet Oncol 16: 804-815, 2015.

40. Liese J, Peveling-Oberhag J, Doering C, Schnitzbauer AA, Herrmann E, Zangos S, Hansmann ML, Moench C, Welker MW, Zeuzem S, et al: A possible role of microRNAs as predictive markers for the recurrence of hepatocellular carcinoma after liver transplantation. Transpl Int 29: 369-380, 2016.

41. Xu LN, Huang DF, Li F, et al: Diagnostic value of serum miRNA-122 and miRNA-221 expression in patients with primary liver cancer. Jiangsu Med J 41: 1285-1288, 2015 (In Chinese).

42. Tan Y, Ge G, Pan T, Wen D, Chen L, Yu X, Zhou X and Gan J: A serum microRNA panel as potential biomarkers for hepatocellular carcinoma related with hepatitis B virus. PLoS One 9: e107986, 2014.

43. Xu J, Wu C, Che X, Wang L, Yu D, Zhang T, Huang L, Li H, Tan W, Wang C and Lin D: Circulating microRNAs, miR-21, miR-122, and miR-223, in patients with hepatocellular carcinoma or chronic hepatitis. Mol Carcinog 50: 136-142, 2011.

44. Qi P, Cheng SQ, Wang H, Li N, Chen YF and Gao CF: Serum microRNAs as biomarkers for hepatocellular carcinoma in Chinese patients with chronic hepatitis B virus infection. PLoS One 6: e28486, 2011.

45. Hsu SH, Wang B, Kota J, Yu J, Costinean S, Kutay H, Yu L, Bai S, La Perle K, Chivukula RR, et al: Essential metabolic, anti-inflammatory, and anti-tumorigenic functions of miR-122 in liver. J Clin Invest 122: 2871-2883, 2012.

46. Wu Q, Liu HO, Liu YD, Liu WS, Pan D, Zhang WJ, Yang L, Fu Q, Xu JJ and Gu JX: Decreased expression of hepatocyte nuclear factor $4 \alpha(\mathrm{Hnf} 4 \alpha) /$ microRNA-122 (miR-122) axis in hepatitis B virus-associated hepatocellular carcinoma enhances potential oncogenic GALNT10 protein activity. J Biol Chem 290 $1170-1185,2015$

47. Li G, Shen Q, Li C, Li D, Chen J and He M: Identification of circulating MicroRNAs as novel potential biomarkers for hepatocellular carcinoma detection: A systematic review and meta-analysis. Clin Transl Oncol 17: 684-693, 2015.

48. He S, Hu XW, Wang D, Han LF, Zhang DC and Wei C: Accuracy of microRNAs for the diagnosis of hepatocellular carcinoma: A systematic review and meta-analysis. Clin Res Hepatol Gastroenterol 40: 405-417, 2016

49. Huang JT, Liu SM, Ma H, Yang Y, Zhang X, Sun H, Zhang X, $\mathrm{Xu} \mathrm{J}$ and Wang J: Systematic review and meta-analysis: Circulating miRNAs for diagnosis of hepatocellular carcinoma. J Cell Physiol 231: 328-335, 2016.

50. Borlado LR and Méndez J: CDC6: From DNA replication to cell cycle checkpoints and oncogenesis. Carcinogenesis 29: 237-243, 2008

51. Deng Y, Jiang L, Wang Y, Xi Q, Zhong J, Liu J, Yang S, Liu R, Wang J, Huang M, et al: High expression of CDC6 is associated with accelerated cell proliferation and poor prognosis of epithelial ovarian cancer. Pathol Res Pract 212: 239-246, 2016.
52. Chen S, Chen X, Xie G, He Y, Yan D, Zheng D, Li S, Fu X, Li Y, Pang X, et al: Cdc6 contributes to cisplatin-resistance by activation of ATR-Chk1 pathway in bladder cancer cells. Oncotarget 7: 40362-40376, 2016.

53. Lee CF, Ling ZQ, Zhao T, Fang SH, Chang WC, Lee SC and Lee KR: Genomic-wide analysis of lymphatic metastasis-associated genes in human hepatocellular carcinoma. World $\mathrm{J}$ Gastroenterol 15: 356-365, 2009.

54. Notas G,Alexaki VI,KampaM,Pelekanou V,Charalampopoulos I, Sabour-Alaoui S, Pediaditakis I, Dessirier V, Gravanis A, Stathopoulos EN, et al: APRIL binding to BCMA activates a JNK2-FOXO3-GADD45 pathway and induces a G2/M cell growth arrest in liver cells. J Immunol 189: 4748-4758, 2012

55. Xiong XD, Fang JH, Qiu FE, Zhao J, Cheng J, Yuan Y, Li SP and Zhuang SM: A novel functional polymorphism in the Cdc6 promoter is associated with the risk for hepatocellular carcinoma. Mutat Res 643: 70-74, 2008

56. Kosakowska-Cholody T, Cholody WM, Hariprakasha HK, Monks AP, Kar SP, Wang M, Michejda CJ and Carr B 1: Growth inhibition of hepatocellular carcinoma cells in vitro and in vivo by the 8-methoxy analog of WMC79. Cancer Chemother Pharmacol 63: 769-778, 2009.

57. Gan N, Du Y, Zhang W and Zhou J: Increase of Mcm3 and Mcm4 expression in cervical squamous cell carcinomas. Eur J Gynaecol Oncol 31: 291-294, 2010.

58. Kikuchi J, Kinoshita I, Shimizu Y, Kikuchi E, Takeda K, Aburatani H, Oizumi S, Konishi J, Kaga K, Matsuno Y, et al: Minichromosome maintenance (MCM) protein 4 as a marker for proliferation and its clinical and clinicopathological significance in non-small cell lung cancer. Lung Cancer 72: 229-237, 2011.

59. Lian M, Fang J, Han D, Ma H, Feng L, Wang R and Yang F: Microarray gene expression analysis of tumorigenesis and regional lymph node metastasis in laryngeal squamous cell carcinoma. PLoS One 8: e84854, 2013.

60. Studach LL, Menne S, Cairo S, Buendia MA, Hullinger RL, Lefrançois L, Merle P and Andrisani OM: Subset of Suz12/PRC2 target genes is activated during hepatitis B virus replication and liver carcinogenesis associated with HBV X protein. Hepatology 56: 1240-1251, 2012.

61. Nan YL, Hu YL, Liu ZK, Duan FF, Xu Y, Li S, Li T, Chen DF and Zeng XY: Relationships between cell cycle pathway gene polymorphisms and risk of hepatocellular carcinoma. World J Gastroenterol 22: 5558-5567, 2016

62. Stolk L, Zhai G, van Meurs JB, Verbiest MM, Visser JA, Estrada K, Rivadeneira F, Williams FM, Cherkas L, Deloukas P, et al: Loci at chromosomes 13,19 and 20 influence age at natural menopause. Nat Genet 41: 645-647, 2009.

63. Cai L, Zhao K and Yuan X: Expression of minichromosome maintenance 8 in chronic myelogenous leukemia. Int J Clin Exp Pathol 8: 14180-14188, 2015.

64. Peng YP, Zhu Y, Yin LD, Zhang JJ, Guo S, Fu Y, Miao Y and Wei JS: The expression and prognostic roles of MCMs in pancreatic cancer. PLoS One 11: e0164150, 2016.

This work is licensed under a Creative Commons Attribution-NonCommercial-NoDerivatives 4.0 International (CC BY-NC-ND 4.0) License. 